\title{
Effects of tanshinone IIA on fibrosis in a rat model of cirrhosis through heme oxygenase-1, inflammation, oxidative stress and apoptosis
}

\author{
MING SHU ${ }^{1}$, XIAO-RONG HU ${ }^{2}$, ZUO-AN HUNG ${ }^{2}$, DAM-DAN HUANG ${ }^{2}$ and SHUN ZHANG ${ }^{2}$ \\ ${ }^{1}$ Department of Hepatobiliary Surgery; ${ }^{2}$ Stem Cell Laboratory, Ningbo No. 2 Hospital, Ningbo, Zhejiang 315010, P.R. China
}

Received March 22, 2015; Accepted November 24, 2015

DOI: $10.3892 / \mathrm{mmr} .2016 .4886$

\begin{abstract}
Tanshinone IIA is extracted from the root of Salvia miltiorrhiza and used in traditional Chinese medicine for its anti-inflammatory activity and antioxidant effects. The aim of the present study was to investigate the potential protective effects of tanshinone IIA against fibrosis in a rat model of cirrhosis and to elucidate the underlying mechanisms. Male Sprague Dawley rats were used as the model of cirrhosis in the present study. In the cirrhotic rats, the extent of fibrosis, levels of alanine aminotransferase (ALT) and aspartate aminotransferase (AST), heme oxygenase-1 (HO-1) protein expression, serum levels of nuclear factor (NF)- $\mathrm{kB}$, tumor necrosis factor- $\alpha$ (TNF- $\alpha$ ), interleukin (IL)-1 $\beta$ and IL-6, superoxide dismutase (SOD), catalase (CAT) and glutathione peroxidase (GSH-PX), and the protein expression levels of phosphorylated-p38 mitogen-activated protein kinase (MAPK) were all significantly increased. However, the serum malondialdehyde (MDA) activity and protein kinase B (Akt) protein expression were suppressed in cirrhotic rats compared with the sham (control) group. Compared with the cirrhotic group, administration of tanshinone IIA reduced the extent of fibrosis, levels of ALT and AST, HO-1 protein expression, serum NF- $\kappa B$, TNF- $\alpha$, IL- $1 \beta$ and IL-6 levels, and the activity of SOD, CAT and GSH-PX. Furthermore, administration of tanshinone IIA significantly increased the inhibition of the serum MDA activity and the Akt protein expression in cirrhotic rats compared with those in the cirrhotic group. The protective effect of tanshinone IIA suppresses fibrosis in a rat model of cirrhosis, and reduces inflammation and oxidative stress, via the HO-1, Akt and p38 MAPK signaling pathway.
\end{abstract}

Correspondence to: Mr. Shun Zhang, Stem Cell Laboratory, Ningbo No. 2 Hospital, 41 Xibei Street, Ningbo, Zhejiang 315010, P.R. China

E-mail: shunzhang2003@163.com

Key words: tanshinone IIA, cirrhotic, heme oxygenase 1, inflammation, oxidative stress, apoptosis

\section{Introduction}

Between 1980 and 2010, liver cirrhosis was associated with the mortality of $\sim 1$ million people and accounted for $2 \%$ of all mortalities worldwide (1). In developed countries, liver cirrhosis is the fifth highest cause of mortality each year (2). Various factors result in liver cirrhosis, however, hepatitis B viral infection and alcohol consumption are the predominant causes. The incidence of cirrhosis varies widely with country, region and age group. Western countries account for more than two-thirds of liver cirrhosis, predominantly resulting from hepatitis B infection, and the incidence is rising each year $(3,4)$.

Heme oxygenase-1 (HO-1) is an enzyme that regulates heme catabolism. The enzyme breaks down hemoglobin or other heme-containing proteins to biliverdin, which is eventually catabolized into bilirubin (5). Release of iron ions in the process generates carbon monoxide (CO). $\mathrm{CO}$ and nitric oxide are important messenger molecules in the body, involved in a variety of pathophysiological processes in disorders of the cardiovascular, respiratory, nervous and digestive systems (6). HO-1 is an inducible heme oxidase, under physiological conditions it is predominantly distributed in the spleen, liver, bone marrow and in the reticuloendothelial system (7). During inflammation, white blood cells such as macrophages, neutrophils and polymorphonuclear cells are observed (8). HO-1 is a type of heat shock protein and expression levels of HO-1 increase in the presence of heavy metal, endotoxins and certain hormones, and under conditions of oxidative stress, inflammation, lack of oxygen, heat shock and cytokine expression (9).

Screening of therapeutic agents from medicinal plants is a major form of novel drug development. Tanshinone IIA is one of the active components of Salvia miltiorrhiza, it has various possible pharmacological effects, in addition to a traditional role in the regulation of menstrual function and removal of pain from blood pooling. Recently, tanshinone IIA has been hypothesized to have a wide array of clinical applications, including antioxidant, antibacterial, and anti-inflammatory effects, in addition to reducing blood viscosity, inhibition of blood coagulation and platelet aggregation, promotion of fibrinolysis, and thrombotic and thrombolytic effects. It protects the liver, exhibits anti-tumor activity, regulates immune function, and increases resistance to diabetic microvascular 
complications (10-12). The present study aimed to investigate whether tanshinone IIA reduces fibrosis in a rat model of cirrhosis via reduced HO-1 activity and reduced inflammation, oxidative stress and apoptosis.

\section{Materials and methods}

Reagents and instruments. The purity of tanshinone IIA (Sigma-Aldrich, St. Louis, MO, USA) was $>97 \%$, and its structure is presented in Fig. 1. Nuclear factor (NF)- $\kappa$ B (ml003404), tumor necrosis factor- $\alpha$ (TNF- $\alpha$; ml001543), interleukin (IL)-1 $\beta$ (ml003549), IL-6 (ml002828), superoxide dismutase (SOD; ml022368), malondialdehyde (MDA; ml016824), catalase (CAT; m1026352) and glutathione peroxidase (GSH-PX; m1026404) enzyme-linked immunosorbent assay (ELISA) kits were obtained from Nanjing KeyGen Biotech. Co., Ltd. (Nanjing, China).

Animals. The experimental procedures were approved by the Animal Care and Use Committee of Ningbo No. 2 Hospital (Ningbo, China). A total of 50 male Sprague Dawley (SD) rats (weight, 250-300 g; age, 8-9 weeks) were obtained from Hangzhou Huaan Biotechnology Co., Ltd. (Hangzhou, China), and were housed in individual cages in an appropriate environment $\left(24 \pm 2^{\circ} \mathrm{C}, 50-70 \%\right.$ humidity, 12 -h light/dark cycle). All rats had ad libitum access to food and water.

Rat model of cirrhosis. SD rats were anesthetized by intraperitoneal injection with $10 \mathrm{mg} / \mathrm{kg}$ xylazine and $100 \mathrm{mg} / \mathrm{kg}$ ketamine hydrochloride (Jiangsu Hengrui Medicine Co., Ltd., Jiangsu, China). Under anesthetic, SD rats underwent double ligation following exposure of the left suprarenal vein. All rats were provided with drinking water with $0.03 \%$ thioacetamide (TAA) solution (Macklin Reagent Co., Ltd., Shanghai, China). The body weight of SD rats was monitored and controlled; if the body weight had increased by 10-20 g following 1 week of treatment with TAA, the concentration of TAA solution was increased by $50 \%$. Conversely, if the body weight of the rats had decreased by 10-20 g after 1 week, the concentration of TAA solution was reduced by $50 \%$. SD rats were continuously treated for 14 weeks.

Grouping. The experimental rats were randomly divided into four groups as follows: i) The sham (control) group $(n=10)$; ii) the cirrhosis group $(\mathrm{n}=10)$; iii) the $10 \mathrm{mg}$ tanshinone IIA group ( $\mathrm{n}=10)$; and iv) the $20 \mathrm{mg}$ tanshinone IIA group $(\mathrm{n}=10)$. In the sham group, healthy rats were administered with physiological saline; in the cirrhosis group, cirrhotic rats were administered physiological saline; in the $10 \mathrm{mg}$ tanshinone IIA group, cirrhotic rats were administered $10 \mathrm{mg} / \mathrm{kg} /$ day of tanshinone IIA for three days; and in the $20 \mathrm{mg}$ tanshinone IIA group, cirrhotic rats were administered with $20 \mathrm{mg} / \mathrm{kg} /$ day of tanshinone IIA for 3 days.

Histological examination. Hematoxylin and eosin (H\&E; ShanghaiQiancheng Biological TechnologyCo.,Ltd.,Shanghai, China) staining was performed according to standard protocols (13). Briefly, following treatment with tanshinone IIA, liver tissue samples (left liver lobe) from all rats were fixed in $0.1 \mathrm{M}$ phosphate buffer (Boster Systems, Inc., Pleasanton,

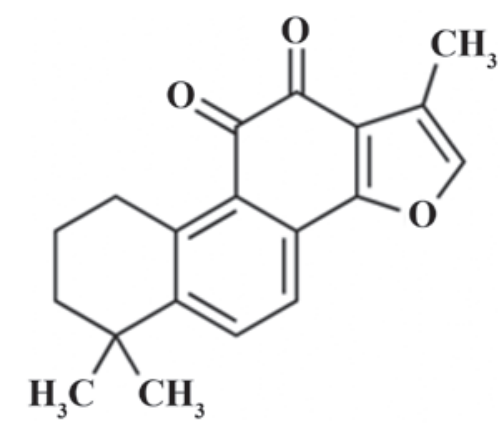

Figure 1. Chemical structure of tanshinone IIA.

CA, USA) containing 4\% paraformaldehyde (Beijing Solarbio Science \& Technology Co., Ltd., Beijing, China) for 12-24 h, and subsequently, in a $30 \%$ sucrose buffer (Shanghai Kang Lang Biological Technology Co., Ltd., Shanghai, China) at room temperature for 2 days. Subsequently, the liver tissue samples were cut into $4-\mu \mathrm{m}$ sections, which underwent $\mathrm{H} \& \mathrm{E}$ staining and were observed under a microscope (HBO 50; Zeiss AG, Oberkochen, Germany).

Measurement of serum alanine aminotransferase (ALT) and aspartate aminotransferase (AST) levels. Following treatment with tanshinone IIA, blood samples were acquired from the caudal veins of the rats at room temperature. The serum ALT and AST levels were analyzed using an AU400 Chemistry Analyzer (Olympus Corporation, Tokyo, Japan) and expressed as international units per liter (U/1).

Western blot analysis. Following treatment with tanshinone IIA, liver tissue samples were homogenized using liquid nitrogen, and lysed with cell lysis buffer (Beyotime Institute of Biotechnology, Haimen, China). The miscible liquids were centrifuged at $4^{\circ} \mathrm{C}$ for $10 \mathrm{~min}$ at $12,000 \mathrm{x} \mathrm{g}$. The supernatant (comprising the total protein) was collected and the protein contents were quantified using a bicinchoninic acid assay (Beyotime Institute of Biotechnology). Equal quantities of protein $(30 \mu \mathrm{g})$ were separated by electrophoresis using 12\% SDS-polyacrylamide gels (Macklin Reagent Co., Ltd.). Following transfer to nitrocellulose filter membranes (Bio-Rad Laboratories, Inc., Hercules, CA, USA) the membranes were blocked with $5 \%$ skimmed milk and incubated with primary antibodies, as follows: Mouse anti-rat HO-1 monoclonal antibody (1:1,000; 374087; EMD Millipore, Billerica, MA, USA), rabbit anti-rat phosphorylated-Akt monoclonal antibody (p-Akt; 1:1,000; 04-802; EMD Millipore), rabbit anti-rat p-p38 mitogen-activated protein kinase monoclonal antibody (p-p38 MAPK; 1:1,000; 09-272; EMD Millipore) and rabbit anti-rat $\beta$-actin polyclonal antibody (1:5,000; BB-2101; BestBio, Inc., Shanghai, China) overnight at $4^{\circ} \mathrm{C}$. Subsequently, the membranes were washed three times for $10 \mathrm{~min}$ each with Tris-buffered saline supplemented with Tween-20 (Beijing Solarbio Science \& Technology Co., Ltd.), prior to incubation with horseradish peroxidase-conjugated goat anti-mouse or anti-rabbit secondary antibodies (1:5,000; BB-2201 and BB-2202; BestBio, Inc.) at $37^{\circ} \mathrm{C}$ for 2-3 h. Finally, the membranes were incubated with chemiluminescence detection substrate for $1 \mathrm{~min}$ (BestBio, Inc.), and visualized using an 

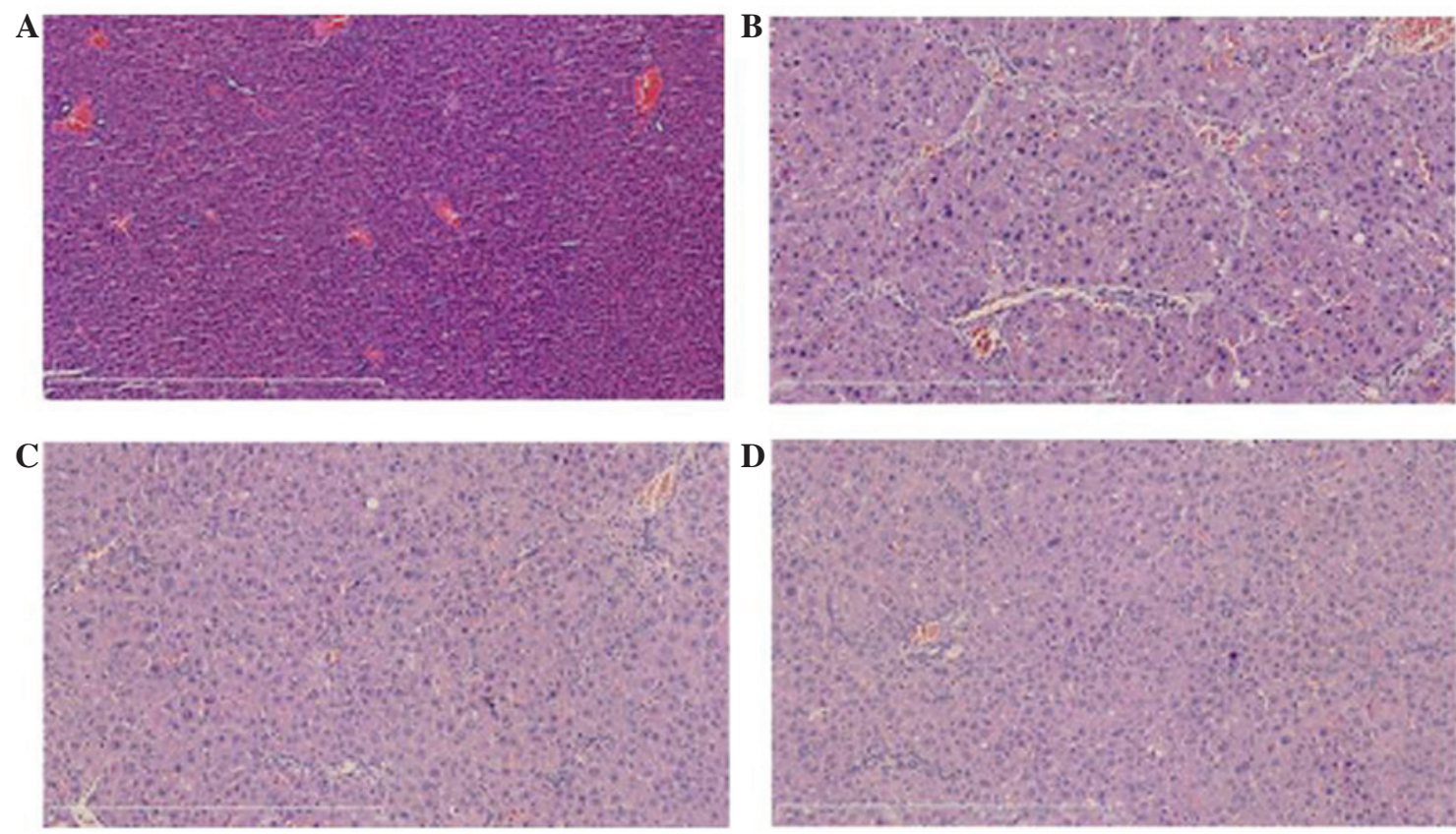

Figure 2. Effects of tanshinone IIA in preventing fibrosis in a rat model of cirrhosis. (A) The sham, (B) cirrhosis, (C) $10 \mathrm{mg}$ tanshinone IIA and (D) $20 \mathrm{mg}$ tanshinone IIA groups. Magnification, x10 in (A) and x50 in (B-D).

A

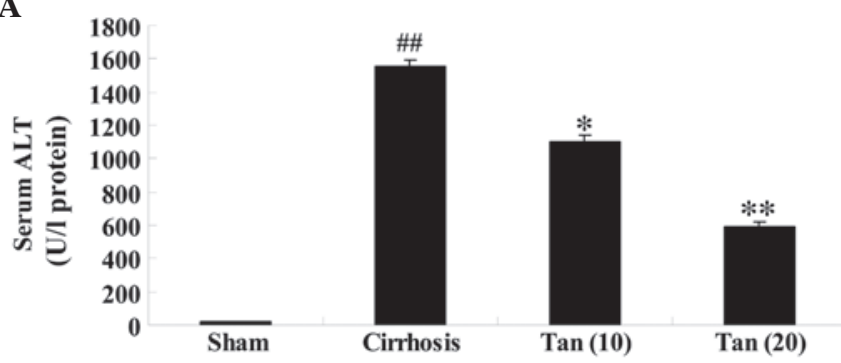

B

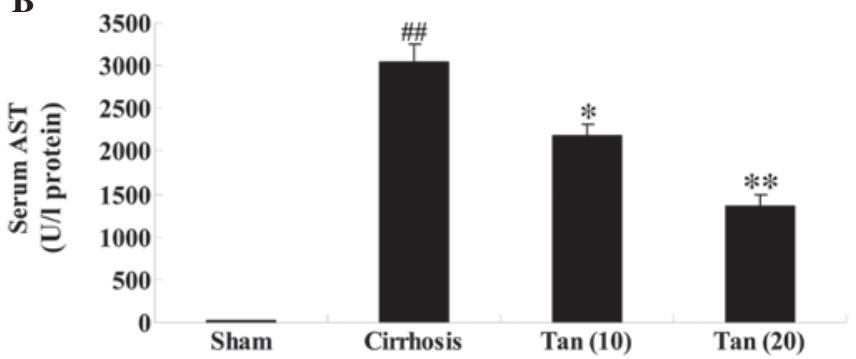

Figure 3. Effects of tanshinone IIA in weakening of the ALT and AST levels in a rat model of cirrhosis. Effects of tanshinone IIA reduce the (A) ALT and (B) AST levels in a rat model of cirrhosis. ${ }^{\# t} \mathrm{P}<0.01$ vs. the sham group; $\mathrm{P}<0.05$ and ${ }^{* * *} \mathrm{P}<0.01$ vs. the cirrhosis group. Tan (10), 10 mg tanshinone IIA group; Tan (20), $20 \mathrm{mg}$ tanshinone IIA group; ALT, alanine aminotransferase; AST, aspartate aminotransferase.

A

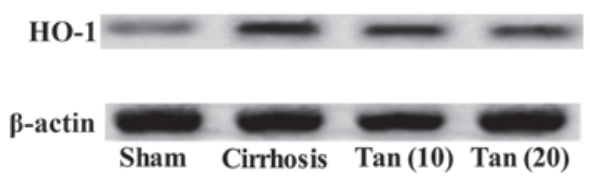

B

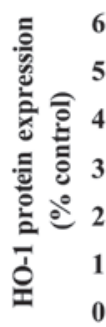

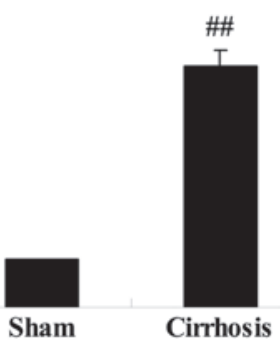

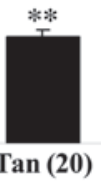

Figure 4. Effects of tanshinone IIA in suppression of HO-1 in a rat model of cirrhosis. (A) Western blot analysis demonstrating HO-1 expression in the rats, and (B) quantification of western blot results in a rat model of cirrhosis. ${ }^{\# \#} \mathrm{P}<0.01$ vs. the sham group; ${ }^{*} \mathrm{P}<0.05$ and ${ }^{* *} \mathrm{P}<0.01$ vs. the cirrhosis group . Tan $(10)$, $10 \mathrm{mg}$ tanshinone IIA group; Tan (20), $20 \mathrm{mg}$ tanshinone IIA group; HO-1, heme oxygenase-1.

enhanced chemiluminescence system (32109; Pierce; Thermo Fisher Scientific, Inc., Waltham, MA, USA). The western blots were analyzed using Quantity One software, version 4.62 (Bio-Rad Laboratories, Inc.)

Measurement of serum NF- $\kappa B, T N F-\alpha, I L-1 \beta$ and IL-6 levels. Following treatment with tanshinone IIA, blood samples were acquired from the caudal vein at room temperature. The serum NF- $\kappa B$, TNF- $\alpha$, IL-1 $\beta$ and IL-6 levels were analyzed using ELISA kits according to the manufacturer's protocols.

Measurement of serum SOD, MDA, CAT and GSH-PX activities. Following treatment with tanshinone IIA, blood samples were acquired from the caudal vein at room temperature. The 
A 60

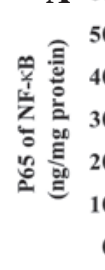

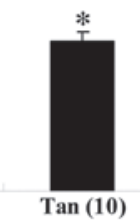
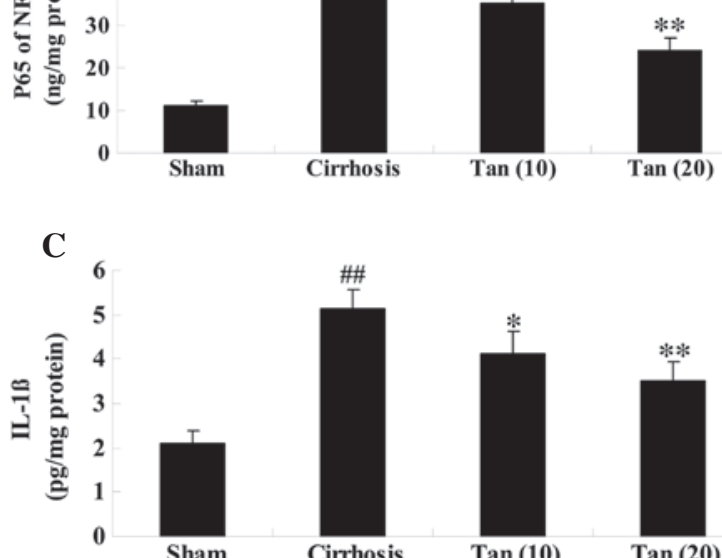
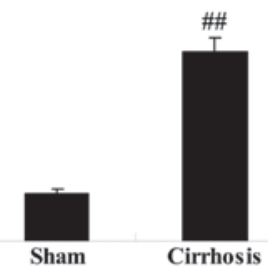

Cirrhosis
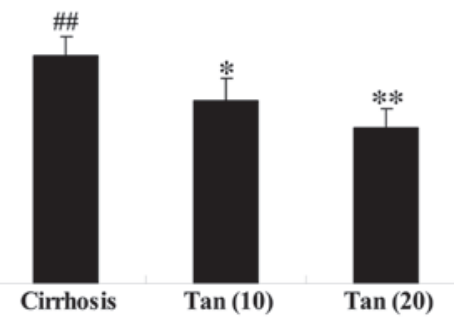

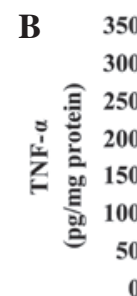

\section{0}

300
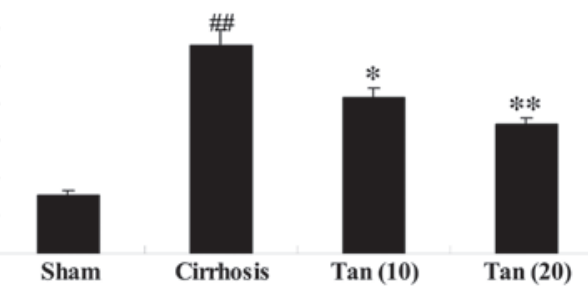

D
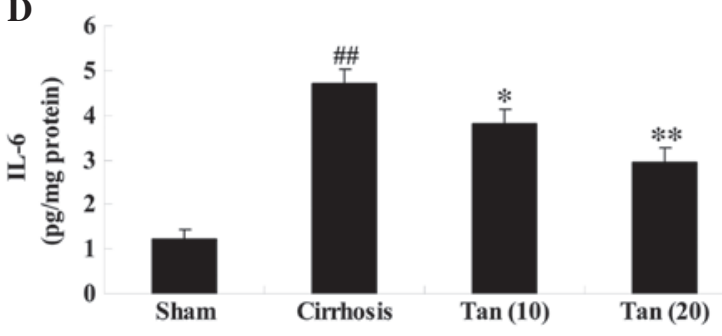

Figure 5. Effects of tanshinone IIA in suppression of inflammation in a rat model of cirrhosis. Tanshinone IIA suppresses the serum levels of (A) NF- $\mathrm{B}$, (B) TNF- $\alpha$, (C) IL-1 $\beta$ and (D) IL-6 in the cirrhotic rats. ${ }^{\# \#} \mathrm{P}<0.01$ vs, the sham group; ${ }^{*} \mathrm{P}<0.05$ and ${ }^{* *} \mathrm{P}<0.01$ vs. the cirrhosis group. Tan (10), $10 \mathrm{mg}$ tanshinone IIA group; Tan (20), 20 mg tanshinone IIA group; NF- $\kappa B$, nuclear factor $\kappa \mathrm{B}$; TNF- $\alpha$, tumor necrosis factor- $\alpha$; IL, interleukin.
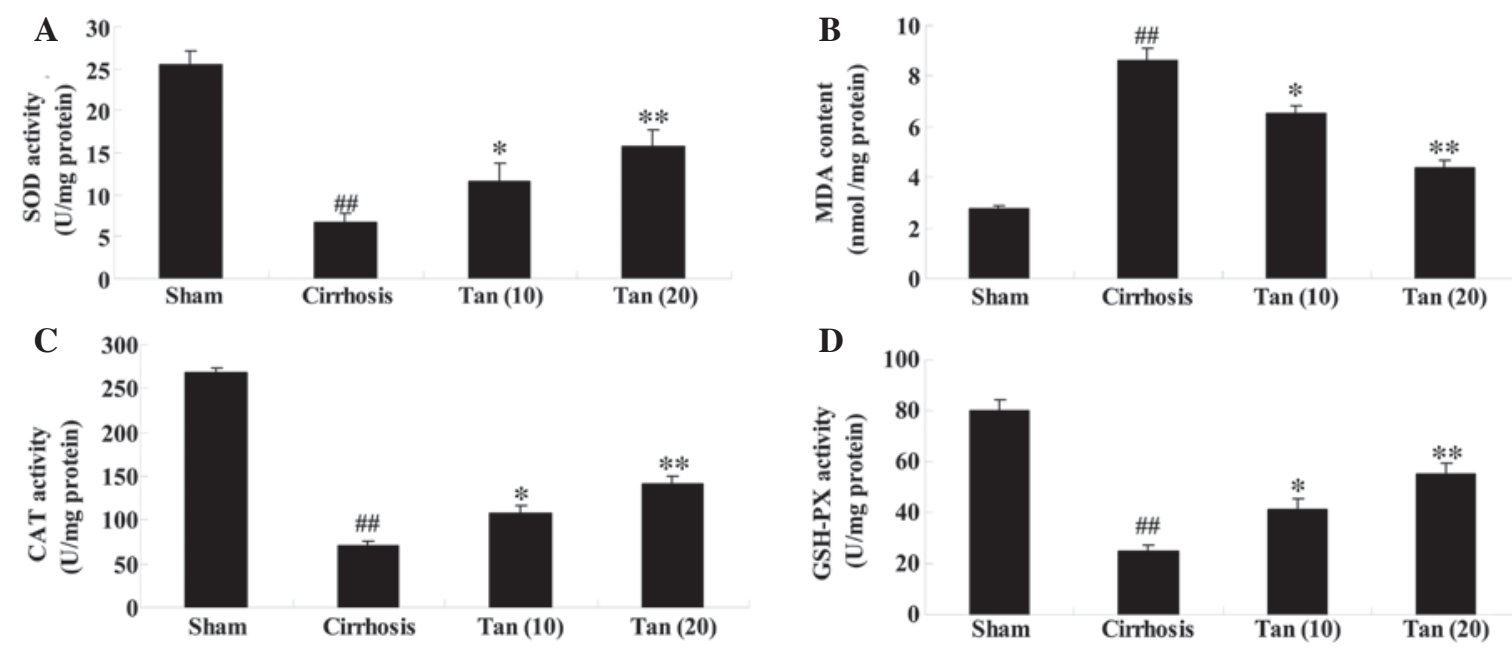

Figure 6. Effects of tanshinone IIA in the suppression of oxidative stress in a rat model of cirrhosis. Tanshinone IIA suppresses the serum levels of (A) SOD, (B) MDA, (C) CAT and (D) GSH-PX in the cirrhotic rats. ${ }^{\# \#} \mathrm{P}<0.01$ vs. the sham group; ${ }^{*} \mathrm{P}<0.05$ and ${ }^{* *} \mathrm{P}<0.01$ vs. the cirrhosis group. Tan (10), $10 \mathrm{mg}$ tanshinone IIA group; Tan (20), 20 mg tanshinone IIA group; SOD, superoxide dismutase; MDA, malondialdehyde; CAT, catalase; GSH-PX, glutathione peroxidase.

serum SOD, MDA, CAT and GSH-PX levels were analyzed using ELISA kits according to the manufacturer's protocols.

Statistical analysis. Data analysis was performed using SPSS software, version 18.0 (SPSS, Inc., Chicago, IL, USA) and expressed as the mean \pm standard deviation. The statistical analysis was performed with analysis of variance. $\mathrm{P}<0.05$ was considered to indicate a statistically significant difference.

\section{Results}

Tanshinone IIA reduced fibrosis in a rat model of cirrhosis. According to the results, the fibrosis was increased in the rat model of cirrhosis (Fig. 2). Compared with the cirrhosis group, the fibrosis was significantly inhibited by treatment with
10 and $20 \mathrm{mg}$ tanshinone IIA $(\mathrm{P}<0.05$ and $\mathrm{P}<0.01$, respectively; Fig. 2).

Tanshinone IIA reduces the levels of ALT and AST in a rat model of cirrhosis. ALT and AST levels were significantly higher in the cirrhosis group, compared with those in the sham group ( $\mathrm{P}<0.01$; Fig. 3). The serum ALT and AST levels were significantly lower in the 10 and $20 \mathrm{mg}$ tanshinone IIA-treated groups compared with the cirrhosis group $(\mathrm{P}<0.05$ and $\mathrm{P}<0.01$, respectively; Fig. 3).

Tanshinone IIA suppresses HO-1 in a rat model of cirrhosis. Compared with the sham group, the cirrhotic rats exhibited significantly elevated HO-1 protein expression levels (Fig. 4). The increase in HO-1 protein expression levels was significantly suppressed by 10 or $20 \mathrm{mg}$ tanshinone IIA treatment, 
$\mathbf{A}$

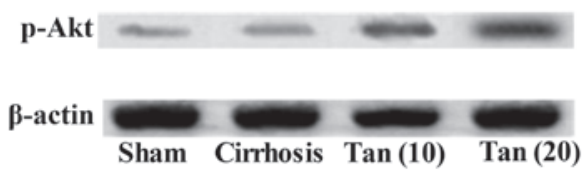

B

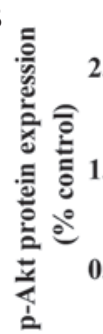

3

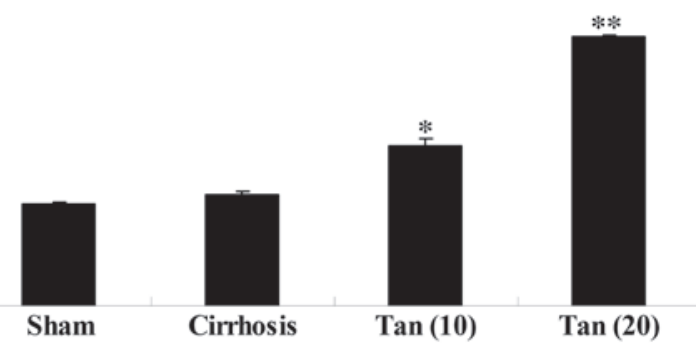

Figure 7. Effects of tanshinone IIA in the activation of Akt in a rat model of cirrhosis. Tanshinone IIA suppressed the p-Akt protein expression assessed by (A) western blotting. (B) Statistical analysis of the p-Akt protein expression. ${ }^{*} \mathrm{P}<0.05$ and ${ }^{* *} \mathrm{P}<0.01$ vs. the cirrhosis group. p-Akt, phosphorylated protein kinase B; Tan (10), $10 \mathrm{mg}$ tanshinone IIA group; Tan (20), $20 \mathrm{mg}$ tanshinone IIA group.

A

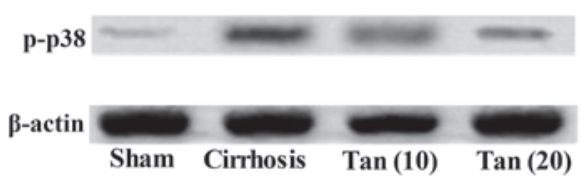

$\mathbf{B}$

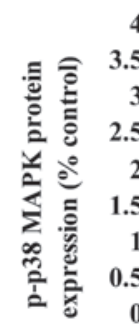

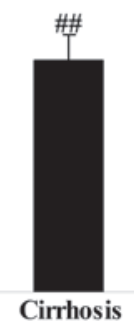
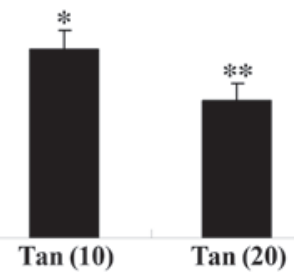

Figure 8. Effects of tanshinone IIA in the suppression of p-38 MAPK in a rat model of cirrhosis. Tanshinone IIA suppressed the p-38 MAPK protein expres-

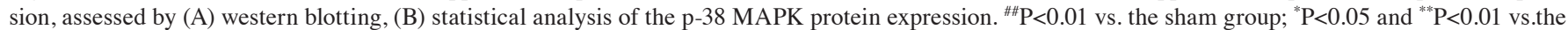
cirrhosis group. Tan (10), $10 \mathrm{mg}$ tanshinone IIA group; Tan (20), $20 \mathrm{mg}$ tanshinone IIA group; MAPK, mitogen-activated protein kinase.

compared with that of the cirrhosis group $(\mathrm{P}<0.05$ and $\mathrm{P}<0.01$, respectively; Fig. 4).

Tanshinone IIA suppresses inflammation in a rat model of cirrhosis. ELISA assays indicated that NF- $\kappa \mathrm{B}, \mathrm{TNF}-\alpha, \mathrm{IL}-1 \beta$ and IL-6 levels were significantly higher in the cirrhotic rats, compared with those in the sham group $(\mathrm{P}<0.01$; Fig. 5$)$. Administration of 10 or $20 \mathrm{mg}$ tanshinone IIA significantly suppressed the serum NF- $\kappa$ B, TNF- $\alpha$, IL- $1 \beta$ and IL- 6 levels in the cirrhotic rats, compared with those of the cirrhosis group $(\mathrm{P}<0.05$ and $\mathrm{P}<0.01$, respectively; Fig. 5).

Tanshinone IIA suppresses oxidative stress in a rat model of cirrhosis. In addition to the increased levels of proteins indicating inflammation in the cirrhotic rats, oxidative stress protein indicators were also observed. Compared with the sham group, the serum SOD, CAT and GSH-PX levels were significantly lower and the serum MDA levels were significantly higher (Fig. 6). Notably, administration of 10 or $20 \mathrm{mg}$ tanshinone IIA significantly reversed these changes compared with the cirrhotic group $(\mathrm{P}<0.05$ and $\mathrm{P}<0.01$, respectively; Fig. 6).

Tanshinone IIA increases Akt in a rat model of cirrhosis. Western blot analysis demonstrated that administration of 10 or $20 \mathrm{mg}$ tanshinone IIA significantly increased the protein expression levels of p-Akt in a rat model of cirrhosis, compared with those of the cirrhosis group $(\mathrm{P}<0.05$ or $\mathrm{P}<0.01$, respectively; Fig. 7).

Tanshinone IIA suppresses p-38 MAPK in a rat model of cirrhosis. As determined by western blot analysis, the protein expression level of p-p38 MAPK in the cirrhotic group was significantly higher than in the sham group (Fig. 8). The protein expression level of p-p38 MAPK was significantly reduced by $10 \mathrm{mg}$ or $20 \mathrm{mg}$ tanshinone IIA treatment, compared with the cirrhosis group $(\mathrm{P}<0.05$ or $\mathrm{P}<0.01$, respectively; Fig. 8).

\section{Discussion}

Liver cirrhosis is a common chronic liver disease resulting from various factors that cause long-term or repeated damage to the liver parenchyma. Its pathological features of diffuse liver fiber hyperplasia, false flocculus and regenerative nodule formation, gradually result in irreversible changes in liver structure, reduced liver function and high pressure in the portal vein (14). Terminal cases present with upper gastrointestinal bleeding, hepatic encephalopathy, ascites and other serious complications (15). In China, liver cirrhosis resulting from viral hepatitis predominates, while ethanol-associated, cardiac and biliary liver cirrhosis are less common, and liver cirrhosis due to genetic metabolic defects is rare (16). The results of the present study demonstrated that the effects of tanshinone IIA ameliorates fibrosis, in addition to reducing ALT and AST levels in a rat model of cirrhosis. Zhang et al (17) demonstrated that tanshinone IIA is protective in lithocholic acid-induced cholestatic liver model. Furthermore, Qi et al (18) also suggested that tanshinone IIA reduces hepatic ischemia-reperfusion. These results indicate that tanshinone IIA may be a novel therapeutic agent for use in cirrhosis.

$\mathrm{HO}$ enzymes are proteases in the microsomes of mammalian cells, there are three heterogeneous types: HO-1, HO-2 and HO-3 (19). Previous studies have demonstrated that HO-1 
is predominantly distributed in the tissue of the reticular endothelial system, the majority of which is in the liver and spleen (20-22). HO is induced by hemoglobin, heavy metals, peroxide, ultraviolet light, oxygen and inflammatory cytokines. The role of HO-1 involves more than the degradation of heme; it also exerts antioxidant and anti-inflammatory effects, as well as dilating blood vessels and possessing anti-apoptotic functions (23). The present study aimed to investigate the effects of tanshinone IIA on HO-1 in a rat model of cirrhosis. A previous study demonstrated that tanshinone IIA inhibited HO-1 expression in RAW 264.7 cells (24). Liu et al (25) also demonstrated that tanshinone IIA reduces the accumulation of cholesterol via the inhibition of HO-1 expression in human macrophages. Results of the present study indicated that the underlying mechanism of tanshinone IIA reducing cirrhosis is associated with the inhibition of HO-1 expression in rats.

Patients with liver cirrhosis present with complex immune system complications. Following infection with a virus, the antigens and antibodies can stimulate mononuclear macrophages that secrete IL-1 and TNF- $\alpha$. High levels of IL-1 and TNF- $\alpha$ induce mononuclear cells and vascular endothelial cells to produce inflammatory cytokines such as IL-8 and IL-6. This activates T cells, B cells, natural killer cells and mononuclear cells. The activation of immunocompetent cells in response to an active infection with a virus leads to changes to the liver cell membrane components, which may dissolve liver cells and lead to necrosis, liver cell damage or abnormal liver function (26). In the present study, pretreatment with tanshinone IIA suppressed inflammation in a rat model of cirrhosis. Wang et al (27) demonstrated that tanshinone IIA suppresses $\mathrm{NF}-\kappa \mathrm{B}$ and apoptosis subsequent to transient middle cerebral artery occlusion. Chen et al (28) suggested that tanshinone IIA protects against cerebral ischemia/reperfusion injury via the release of TNF- $\alpha$ and IL- 6 in rats.

Oxidative stress is considered to be an important factor in liver damage. Oxidative stress refers to free radicals and reactive oxygen species damaging proteins, nucleic acids and lipids in a macromolecular reaction, resulting in a change in the state of the structure and loss of function (29-31). A previous study reported that oxidative stress is closely associated with liver cell damage (32). SOD is the predominant defensive enzyme in the antioxidant system of the body. It catalyzes a disproportionation reaction of the superoxide anion, to prevent tissue damage $(33,34)$. GSH is an effective free radical scavenger that inhibits lipid peroxidation and MDA is the end product of the cell membrane lipid peroxidation chain (35). Thus, the presence of this indicator in liver cells suggests oxidative stress. Continuous and irreversible oxidative stress results in liver cell membrane lipid peroxidation and cell dysfunction, eventually leading to cell death (36). SOD, GSH and MDA are utilized to evaluate the oxidative stress levels in a cell. SOD and GSH reflect the ability of the cells to clear oxygen free radicals, while MDA reflects the severity of free radical attack (37). In the current study, tanshinone IIA suppressed oxidative stress in a rat model of cirrhosis. Chan et al (38) suggested that tanshinone IIA weakens $\mathrm{H}_{2} \mathrm{O}_{2}$-induced vein endothelial cell injury. Zhang et al (39) reported that tanshinone IIA attenuates oxidative stress injury in kidney hypothermic preservation. The results of these studies suggested that tanshinone IIA has a protective effect against inflammation and oxidative stress in a rat model of cirrhosis.

Hepatocellular apoptosis is an important factor in the development of liver cirrhosis, healthy liver development and in processes of a variety of liver diseases; when the liver is damaged, a pre-existing liver cell death program is activated, liver cell apoptosis increases, producing a large number of apoptotic bodies (40). Following apoptosis, hepatic stellate cells phagocytoze the cellular material and are activated to produce large quantities of extracellular matrix and collagen, eventually resulting in the formation of liver fibrosis $(41,42)$. Therefore, pathological liver cell apoptosis is closely associated with liver fibrosis (43). The results suggest that tanshinone IIA suppresses cell apoptosis in a rat model of cirrhosis via the activation of Akt and inhibition of p38 MAPK. Luo et al (44) hypothesized that the anti-remodeling property of tanshinone IIA reduces cell apoptosis via the Akt/S-phase kinase-associated protein 2/p27-associated signaling pathway in hypoxia-induced pulmonary artery smooth muscle cell proliferation. Jang et al (45) suggested that lipopolysaccharide-induced $\mathrm{NF}-\kappa \mathrm{B}$ activation is reduced by treatment with tanshinone IIA via the suppression of the MAPKs (p38, extracellular signal-related kinase $1 / 2$ and c-Jun N-terminal kinase) signaling pathway.

In conclusion, the present study demonstrated the protective effect of tanshinone IIA in reducing fibrosis, reduced liver injury, reducing HO-1 expression, and exerting anti-inflammatory, anti-oxidative and anti-apoptotic effects in a rat model of cirrhosis.

\section{Acknowledgements}

The present study was funded by the Zhejiang Provincial Natural Science Foundation of China (grant no. Y2080096), the Ningbo Science and Technology Innovation Team Project (grant no. 2011B82016), the Ningbo Natural Science Foundation (grant no. 2007A610063) and the Zhejiang Province Medical Science Research Project (grant no. 2007A172).

\section{References}

1. Mokdad AA, Lopez AD, Shahraz S, Lozano R, Mokdad AH, Stanaway J, Murray CJL and Naghavi M: Liver cirrhosis mortality in 187 countries between 1980 and 2010: A systematic analysis. BMC Med 12: 145, 2014.

2. Ciećko-Michalska I, Wójcik J, Senderecka M, Wyczesany M, Binder M, Szewczyk J, Dziedzic T, Słowik A and Mach T: Cognitive functions in patients with liver cirrhosis: A tendency to commit more memory errors. Med Sci Monit 19: 283-288, 2013.

3. Matsuda I, Okada M, Inoue T, Tokugawa T, Ogawa H and Hirota S: Primary follicular lymphoma of the spleen incidentally found in a patient with alcohol- and hepatitis C-related liver cirrhosis. Int J Clin Exp Pathol 7: 4484-4488, 2014.

4. Wang X, Xie G, Wang X, Zhou M, Yu H, Lin Y, Du G, Luo G and Liu P: Urinary metabolite profiling offers potential for differentiation of liver-kidney yin deficiency and dampness-heat internal smoldering syndromes in posthepatitis B cirrhosis patients. Evid Based Complement Alternat Med 2015: 464969, 2015.

5. Deng C, Tao R, Yu SZ and Jin H: Sulforaphane protects against 6-hydroxydopamine-induced cytotoxicity by increasing expression of heme oxygenase-1 in a PI3K/Akt-dependent manner. Mol Med Rep 5: 847-851, 2012.

6. Chakravarti R, Gupta K, Majors A, Ruple L, Aronica M and Stuehr DJ: Novel insights in mammalian catalase heme maturation: EFFect of NO and thioredoxin-1. Free Radic Biol Med 82: 105-113, 2015. 
7. Bao LJ, Jaramillo MC, Zhang ZB, Zhang YX, Yao M, Zhang DD and Yi XF: Nrf2 induces cisplatin resistance through activation of autophagy in ovarian carcinoma. Int J Clin Exp Pathol 7 : $1502-1513,2014$

8. Yang D, Zhang W, Song L and Guo F: Andrographolide protects against cigarette smoke-induced lung inflammation through activation of heme oxygenase-1. J Biochem Mol Toxicol 27: 259-265, 2013.

9. Fauconneau B, Petegnief V, Sanfeliu C, Piriou A and Planas AM: Induction of heat shock proteins (HSPs) by sodium arsenite in cultured astrocytes and reduction of hydrogen peroxide-induced cell death. J Neurochem 83: 1338-1348, 2002.

10. Gao S,Liu Z,Li H,Little PJ,Liu Pand Xu S: Cardiovascular actions and therapeutic potential of tanshinone IIA. Atherosclerosis 220: $3-10,2012$.

11. Tian $\mathrm{XH}$ and $\mathrm{Wu} \mathrm{JH}$ : Tanshinone derivatives: A patent review (January 2006 - September 2012). Expert Opin Ther Pat 23 19-29, 2013.

12. Xu S and Liu P: Tanshinone II-A: New perspectives for old remedies. Expert Opin Ther Pat 23: 149-153, 2013.

13. Prent $\varnothing$ P: Van Gieson's picrofuchsin. The staining mechanisms for collagen and cytoplasm, and an examination of the dye diffusion rate model of differential staining. Histochemistry 99: $163-174,1993$

14. Kawanaka H, Akahoshi T, Itoh $\mathrm{S}$, Iguchi $\mathrm{T}$, Harimoto $\mathrm{N}$ Uchiyama H, Yoshizumi T, Shirabe K, Takenaka K and Maehara Y: Optimizing risk stratification in portal vein thrombosis after splenectomy and its primary prophylaxis with antithrombin III concentrates and danaparoid sodium in liver cirrhosis with portal hypertension. J Am Coll Surg 219: 865-874, 2014.

15. Yu F, Lin Z, Zheng J, Gao S, Lu Z and Dong P: Suppression of collagen synthesis by Dicer gene silencing in hepatic stellate cells. Mol Med Rep 9: 707-714, 2014.

16. Iwaisako K, Hatano E, Taura K, Nakajima A, Tada M, Seo S Tamaki N, Sato F, Ikai I, Uemoto S and Kinoshita M: Loss of Sept 4 exacerbates liver fibrosis through the dysregulation of hepatic stellate cells. J Hepatol 49: 768-778, 2008.

17. Zhang X, Ma Z, Liang Q, Tang X, Hu D, Liu C, Tan H, Xiao C, Zhang B, Wang Y and Gao Y: Tanshinone IIA exerts protective effects in a LCA-induced cholestatic liver model associated with participation of pregnane X receptor. J Ethnopharmacol 164 357-367, 2015

18. Qi YY, Xiao L, Zhang LD, Song SH, Mei Y, Chen T, Tang JM, Liu F, Ding GS, Shi YZ and Wang QX: Tanshinone IIA pretreatment attenuates hepatic ischemia-reperfusion. Front Biosci (Elite Ed) 4: 1303-1313, 2012.

19. Agca CA, Tuzcu M, Hayirli A and Sahin K: Taurine ameliorates neuropathy via regulating $\mathrm{NF}-\kappa \mathrm{B}$ and $\mathrm{Nrf} 2 / \mathrm{HO}-1$ signaling cascades in diabetic rats. Food Chem Toxicol 71: 116-121, 2014.

20. Chen Y, Huang F, Wang D, Weng Z and Deng Z: Upregulation of heme oxygenase-1 expression may facilitate memory and learning in mice. Exp Ther Med 5: 1491-1495, 2013.

21. Wunder C and Potter RF: The heme oxygenase system: Its role in liver inflammation. Curr Drug Targets Cardiovasc Haematol Disord 3: 199-208, 2003.

22. Hachfi L, Simide R, Richard S, Couvray S, Coupe S, Gaillard S, Pierre S, Grillasca JP and Prevot-D'Alvise N: Effect of water temperature increase on HO-1 expression in European sea bass (Dicentrarchus labrax L.) tissues. Cell Mol Biol (Noisy-le-grand) Suppl.58: OL1752-OL1756, 2012.

23. Li H, Zhou $X$ and Zhang J: Induction of heme oxygenase-1 attenuates lipopolysaccharide-induced inflammasome activation in human gingival epithelial cells. Int J Mol Med 34: 1039-1044, 2014.

24. Chen TH, Hsu YT, Chen CH, Kao SH and Lee HM: Tanshinone IIA from Salvia miltiorrhiza induces heme oxygenase-1 expression and inhibits lipopolysaccharide-induced nitric oxide expression in RAW 264.7 cells. Mitochondrion 7: 101-105, 2007.

25. Liu Z, Wang J, Huang E, Gao S, Li H, Lu J, Tian K, Little PJ, Shen X, Xu S and Liu P: Tanshinone IIA suppresses cholesterol accumulation in human macrophages: Role of heme oxygenase-1. J Lipid Res 55: 201-213, 2014.
26. Barak V, Selmi C, Schlesinger M, Blank M, Agmon-Levin N, Kalickman I, Gershwin ME and Shoenfield Y: Serum inflammatory cytokines, complement components, and soluble interleukin 2 receptor in primary biliary cirrhosis. J Autoimmun 33: 178-182, 2009.

27. Wang JG, Bondy SC, Zhou L, Yang FZ, Ding ZG, Hu Y, Tian Y, Wen PY, Luo H, Wang F, et al: Protective effect of Tanshinone IIA against infarct size and increased HMGB1, NFкB, GFAP and apoptosis consequent to transient middle cerebral artery occlusion. Neurochem Res 39: 295-304, 2014.

28. Chen Y, Wu X, Yu S, Lin X, Wu J, Li L, Zhao J and Zhao Y Neuroprotection of tanshinone IIA against cerebral ischemia/reperfusion injury through inhibition of macrophage migration inhibitory factor in rats. PLoS One 7: e40165, 2012.

29. Apel K and Hirt H: Reactive oxygen species: metabolism, oxidative stress, and signal transduction. Annu Rev Plant Biol 55: 373-399, 2004.

30. McCord JM: The evolution of free radicals and oxidative stress. Am J Med 108: 652-659, 2000

31. Mittler R: Oxidative stress, antioxidants and stress tolerance. Trends Plant Sci 7: 405-410, 2002.

32. Szuster-Ciesielska A, Daniluk J and Kandefer-Szerszeń M: Oxidative stress in the blood of patients with alcohol-related liver cirrhosis. Med Sci Monit 8: CR419-424, 2002.

33. Medina J and Moreno-Otero R: Pathophysiological basis for antioxidant therapy in chronic liver disease. Drugs 65: 2445-2461, 2005.

34. Koch OR, Pani G, Borrello S, et al.: Oxidative stress and antioxidant defenses in ethanol-induced cell injury. Mol Aspects Med 25: 191-198, 2004.

35. Patel SN, Pandya K, Clark GJ, Parikh MC and Lau-Cam CA: Comparison of taurine and pantoyltaurine as antioxidants in vitro and in the central nervous system of diabetic rats. Exp Toxicol Pathol (In press).

36. Li S, Tan HY, Wang N, et al.: The Role of Oxidative Stress and Antioxidants in Liver Diseases. Int J Mol Sci 16: 26087-26124, 2015.

37. Oettl K, Stadlbauer V, Petter F, Greilberger J, Putz-Bankuti C, Hallström S, Lackner C and Stauber RE: Oxidative damage of albumin in advanced liver disease. Biochim Biophys Acta: 469-473, 2008

38. Chan P, Chen YC, Lin LJ, Cheng TH, Anzai K, Chen YH, Liu ZM, Lin JG and Hong HJ: Tanshinone IIA attenuates $\mathrm{H}_{2} \mathrm{O}_{2}$-induced injury in human umbilical vein endothelial cells. Am J Chin Med 40: 1307-1319, 2012

39. Zhang X, He D, Xu L and Ling S: Protective effect of tanshinone IIA on rat kidneys during hypothermic preservation. Mol Med Rep 5: 405-409, 2012.

40. Shin DY, Kim GY, Kim ND, Jung JH, Kim SK, Kang HS and Choi YH: Induction of apoptosis by pectenotoxin-2 is mediated with the induction of DR4/DR5, Egr-1 and NAG-1, activation of caspases and modulation of the Bcl-2 family in p53-deficient Hep3B hepatocellular carcinoma cells. Oncol Rep 19: 517-526, 2008.

41. Lambrecht J, Mannaerts I and van Grunsven LA: The role of miRNAs in stress-responsive hepatic stellate cells during liver fibrosis. Front Physiol 6: 209, 2015.

42. Zhou WC, Zhang QB and Qiao L: Pathogenesis of liver cirrhosis. World J Gastroenterol 20: 7312-7324, 2014.

43. Park ST, Jang JW, Kim GD, Park JA, Hur W, Woo HY, Kim JD, Kwon JH, Yoo CR, Bae SH: Beneficial effect of metronomic chemotherapy on tumor suppression and survival in a rat model of hepatocellular carcinoma with liver cirrhosis. Cancer Chemother Pharmacol 65: 1029-1037, 2010.

44. Luo Y, Xu DQ, Dong HY, Zhang B, Liu Y, Niu W, Dong MQ and Li ZC: Tanshinone IIA inhibits hypoxia-induced pulmonary artery smooth muscle cell proliferation via Akt/Skp2/p27-associated pathway. PLoS One 8: e56774, 2013.

45. Jang SI, Kim HJ, Kim YJ, Jeong SI and You YO: Tanshinone IIA inhibits LPS-induced NF-kappaB activation in RAW 264.7 cells: Possible involvement of the NIK-IKK, ERK1/2, p38 and JNK pathways. Eur J Pharmacol 542: 1-7, 2006. 